\title{
Reconfiguration of distribution system with distributed generation using an adaptive loop approach
}

\author{
Darko Šošić, Predrag Stefanov
}

\begin{abstract}
A new concept of switches selection in the meta-heuristic optimization process of optimal distribution network reconfiguration has been proposed. Based on the adaptive set of selectable candidates, the proposed concept determines the switch status. This approach prevents the creation of unfeasible solutions (non-radial and unconnected configurations), and significantly reducing the number of searches and accelerating the optimization process. Unfeasible solutions, created by meta-heuristic optimization rules, can be corrected by means of the proposed adaptive loop concept. The correct parts of the unfeasible solution are retained, while only the defective parts are replaced by the adaptively formed loops from the currently available conditions that respect the correct switching operations. In this way, the basic characteristics of the optimization process have been retained to the greatest possible extent. Tests were performed on a two different size standard distribution networks.

K e y w or d s: distribution systems, reconfiguration, load flow, optimization
\end{abstract}

\section{Introduction}

The reconfiguration of the distribution network is one of the most important management functions that optimize the operation of distribution networks. Optimal reconfiguration ensures optimal status of switching devices from the aspect of different, user-defined optimization criteria. These optimization criteria are usually related to minimizing the loss of real power in distribution network, balancing the load on branches of network, and reliability indices of consumers supply. The optimal solution must fulfil the thermal limitations related to the elements of the distribution network, as well as provide the appropriate voltage for all consumers. A radial configuration of the distribution network is preferred in order to facilitate the easy adjustment of relay protection and regulation devices, irrespective of the presence or absence of connected distributed sources.

As such, the reconfiguration problem is classified as a mixed integer/binary nonlinear non-differentiable constrained optimization problem. Instead of the classical optimization algorithms $[1,2]$, a series of heuristic methods have been proposed to solve this problem [3-6]. In [6] a new heuristic approach is proposed for reconfiguration of a distribution network in the presence of distributed generation. The heuristic algorithms are very fast to solve the reconfiguration problem and are appropriate for real time distribution automation. However, the solutions of heuristic methods strongly depend on the initial configuration of the network. While on the other hand, metaheuristic methods enable better, spread search of the so- lution space and, depending on the applied method, they can find solution that is close enough to the global optimum. Therefore, meta-heuristic methods have been used more frequently in recent years for solving this problem: ant colony algorithm [7], gray wolf optimizer [8], particle swarm optimization [9], cuckoo search algorithm [10], genetic algorithm [11], etc. In addition to meta-heuristic methods, in recent years, the number of papers utilizing other artificial intelligence tools such as fuzzy logic and clustering algorithms $[12,13]$ has also increased. In [14], the authors proposed parallel-based genetic algorithm for network reconfiguration in power system planning whereas in [15], they put forward a mixed approach of heuristic and meta-heuristic method. A method for load restoration after natural disasters considering coordination of system reconfiguration and creation of separate microgrids using mixed-integer second-order cone programming was proposed in [16]. These methods were used in single-criterion or multi-objective optimization algorithms. In [17], the fast NSGA was used for minimization of real power losses thereby improving voltage profile and load balancing index with minimum switching operations. Hybrid optimization algorithm combining the concept of fuzzy Pareto dominance with shuffled frog leaping algorithm aimed at recognizing optimal non-dominated solutions is presented in [18]. A multi-objective gravitational search algorithm for distribution feeder reconfiguration with distributed generators was proposed in [19]. After solving multi-objective problem and obtaining Pareto front, the most preferred solution is chosen using a fuzzy decision-making tool.

* University of Belgrade, School of Electrical Engineering, Bulevar kralja Aleksandra 73, 11000 Belgrade, Serbia, sosic@etf.rs 
Although these methods focus on the problems of criterion function non-differentiability and the premature stopping in local optimum, new problems are encountered since they are mostly combinatorial methods by default. These problems are mainly associated with a large number of searches, and with a significant number of discarded unfeasible solutions. The reason for solution rejection can be violation of the allowed limits of control variables (real numbers), or creation of non-radial or unconnected configurations (integer/binary numbers). Consequently, this creates space for further improvement of these methods.

Regardless of the optimization methods in many of the above-mentioned papers the fundamental loop concept was used [20]. Fundamental loops are used to reduce the number of created unfeasible solutions. This concept represents a major advancement regarding binary coding [21], but there is a serious deficiency that is reflected in the possibility of forming islands or unconnected parts of the network. If an unfeasible solution is generated during an optimization process, it is discarded and the new one is created instead. This is the main reason why a new approach of branch coding has been proposed in this paper, and it has been dubbed the adaptive loop concept.

Based on the fundamental loop concept, the proposed procedure determines the solutions based on the adaptive selection of loops. In other words, aimed at ensuring the radial structure of network, a selection of switches is being performed successively for each control variable thus preventing the creation of an unconnected network. This greatly reduces the number of searches and accelerates the process of generating the population. It is possible to correct unfeasible solutions that are created with meta-heuristic optimization rules by utilizing the proposed adaptive loop concept. Furthermore, with this concept, from every unfeasible solution, the maximum of correct data is extracted, and only the bad data set is corrected. In this way, the obtained corrected solution is the closest to the considered unfeasible solution, by which the basic characteristics of the selected optimization process remain unchanged. Therefore, using the adaptive loop concept prevents rejection of irregular solutions that are generated using the operators of optimization algorithm. The total calculation time decrease is exactly what occurs caused by this, since the corrected solution is compared with the already existing solution in the "knockout" selection stage and, therefore, only better one goes to the next population.

In distribution network real-time measurements are limited and network observability is not achieved unless pseudo measurements are used. Pseudo measurements of active and reactive power injections and consumptions are based on forecasted and historical data. According to [22], methods for load forecasting can be deterministic and probabilistic, depending on chosen level of uncertainty. In this paper, probabilistic fuzzy representation of power injections and consumptions are used. For these reasons, the authors used the previously developed fuzzy load flow [23].
Adaptive loop concept has been implemented and tested by means Differential Evolution (DE) [24], which proved to be an excellent choice when solving some other power systems problems $[25,26]$. The testing of the optimization process was carried out on the two standard test distribution networks (IEEE 33 and 119-bus system) with controllable switches in all branches. The reduction of real power losses in the network with/without distributed production is considered in proposed algorithm.

After the introductory considerations in which the literature has been reviewed and the main idea of this paper presented, we propose the general formulation of the optimization problem of distribution network reconfiguration, focusing on the concept of adaptive loops for the creation of the initial population and the correction of unfeasible solutions. After the applied optimization algorithm is described we give a complete overview of the used optimization and present the simulation results of proposed method on IEEE 33-bus and IEEE 119-bus test systems.

\section{Mathematical problem}

The optimal reconfiguration provides status of distribution network switching equipment in terms of different optimization criteria. In order to create a connected radial configuration of the distribution network with the optimal value of the considered criteria function, it is necessary to find a set of control variables related to switching elements status that need to be opened in a given operating mode (or in a certain time interval) in this process. In order to simplify presentation of the proposed algorithm a static problem is considered, related to a single operating regime with a given load and production. In this paper, the reduction of real power losses is considered

$$
F=\min \sum_{i=1}^{N_{b}} R_{i} J_{i}^{2}
$$

where $R_{i}$ is resistance of the $i$-th branch, $N_{b}$ is the number of branches of the observed distribution network, $J_{i}$ represents the current that flow through the $i$-th branch.

\subsection{Constraints}

The generated distribution network configuration must overcome the imposed topological and operational constraints that system operator has to consider when modifying the distribution network. These constraints are current flow limits through branches, voltage deviation limits, network connectivity and radial operation. The last stated constraint occurs due to technical reasons, such as voltage regulation and simplification of protection schemes. The feasible solutions are those that fulfil the next set of constraints:

1) Power flow equations as equality constraints

$$
J_{i}=I_{i}+\sum_{q \in \alpha} J_{q}
$$


2) Branch capacity constraints

$$
\left|J_{i, \min }\right| \leq\left|J_{i}\right| \leq\left|J_{i, \max }\right|, \quad i=1, \ldots, N_{b} .
$$

3) Node voltage constraints

$$
\left|V_{j, \min }\right| \leq\left|V_{j}\right| \leq\left|V_{j, \max }\right|, \quad j=1, \ldots, N_{\text {bus }} .
$$

4) All buses are supplied

$$
\operatorname{loop}(\boldsymbol{A})=\boldsymbol{0} .
$$

5) Radial network structure is maintained [8]

$$
\operatorname{det}\left(\boldsymbol{A}_{1}\right)=1 \quad \text { or }-1
$$

Above, $I_{i}$ is load current in the receiving node of the $i$ th branch, $\alpha$ is the set of all downstream branches that are connected to the $i$-th node, $J_{i, \min }$ and $J_{i, \max }$ are lower and upper limit of the line current of $i$-th branch, respectively. $V_{j}$ is voltage of $j$-th bus, while $V_{j \text {,min }}$ and $V_{j, \max }$ are lower and upper limit of $j$-th bus voltage, respectively. $\boldsymbol{A}$ is connection matrix which has one row for each branch and one column for each node. In this matrix if branch $i$ is directed from node $j$, element $A_{i j}$ is equal to 1 , and if it is directed toward node $j$, element $A_{i j}$ is equal to -1 , otherwise element $A_{i j}$ is zero. The loop is iterative function that examines if the considered network is connected. In each iteration this function calculates absolute sum of each element of a corresponding column in matrix $\boldsymbol{A}$, and nodes whose sum is equal to 1 determine which branch (row) will be removed from the matrix $\boldsymbol{A}$ [8]. The iterative procedure ends when there are no more nodes whose sum is equal to 1 . There are two possible outputs from the loop function. In the first case, matrix $\boldsymbol{A}$ is not empty and the remaining branches form a loop, while in the other case an empty set indicates that the network is connected. Since there is $N_{b}=N_{\text {bus }}-1$ branches in the network, if there is a loop in a network, one node is left without supply. $\boldsymbol{A}_{1}$ is connection matrix, which is the same as matrix $\boldsymbol{A}$ where the first column corresponding to the reference node in the network is removed.

\section{Adaptive loop concepts}

In the optimal reconfiguration problem, the number of control variables is equal with the number of switching elements. By simultaneously changing the status of switching elements, the network configuration changes in order to optimize the criteria function. Developed heuristic methods generally allow changing the status of only one pair of switching elements [4,27]. Using this procedure, the created solution is very dependent on the initial state of the distribution network and generally does not allow finding of global optimum. Simultaneous consideration of many switching actions makes the reconfiguration problem extremely difficult, which was the reason for the introduction of meta-heuristic methods in solving this problem. The necessity of creating a radial structure and supply of all consumers further complicates this problem, since in many cases the configurations that have loops or unconnected customers could be created.

Great progress in this area has been achieved by applying the fundamental loops concept [20]. Assuming, that an available switching device exists in each branch of the distribution network, the number of control variables has been reduced to the number of independent - fundamental loops of the network formed according to the positions of normally open switches in the initial configuration. The possibility of switch status change is related to determination of their belonging to individual fundamental loop. Since some branches belong to one or more fundamental loops, the choice of control variables is not independent, as is usually adopted in the application of this concept. However, this does not prevent the possibility of forming islands or isolating a group of consumers. Additionally, this is the main disadvantage of this approach. Generated unfeasible solutions are then rejected and the procedure is repeated until a feasible solution has been obtained.

With the adaptive loop concept solutions with loops or unconnected configuration of distribution network are avoided. The choice of control variables is related to the successive analysis of the loops that change depending on the variables chosen up to then. This procedure can be used in the initial population formation as well as the unfeasible solution correction obtained using the optimization operator. A detailed overview of the necessary steps for these two cases is given in the following sections.

\subsection{Creation of the initial population}

As stated above, sets of branches are not unchangeable. Yet, they change with every change in the network configuration. In order to simplify the explanation of the adaptive loops concept, the distribution network configuration will be represented by an oriented graph. The direction of the oriented branch goes from the sending end to the receiving end, ie it coincides with the natural power flow of the radial distribution networks and is uniquely determined for each feasible configuration. In addition, two operators " $\leftarrow$ " and " $\rightarrow$ " have been introduced, which are used to define the search direction and to collect the allowed candidates for individual control variables. The first operator " $\leftarrow$ " indicates that the search and the addition of a branch into the corresponding set is done in opposite direction from the orientation of the network branches, while the other operator " $\rightarrow$ " indicates that the search is done in the same direction.

Each solution $a$ is defined by a vector of control variables $\boldsymbol{t}_{a}$. Elements of this vector, $\boldsymbol{t}_{a}=\left[t_{a 1}, \ldots, t_{a e}\right]$, are the indexes of those network branches in which switching equipment is in the normally open state. All other switching elements are in the closed position. In order to enable the radial configuration, the length of the control vector is determined by the number of the fundamental loops of the network $-e$. The algorithm for creating the initial 


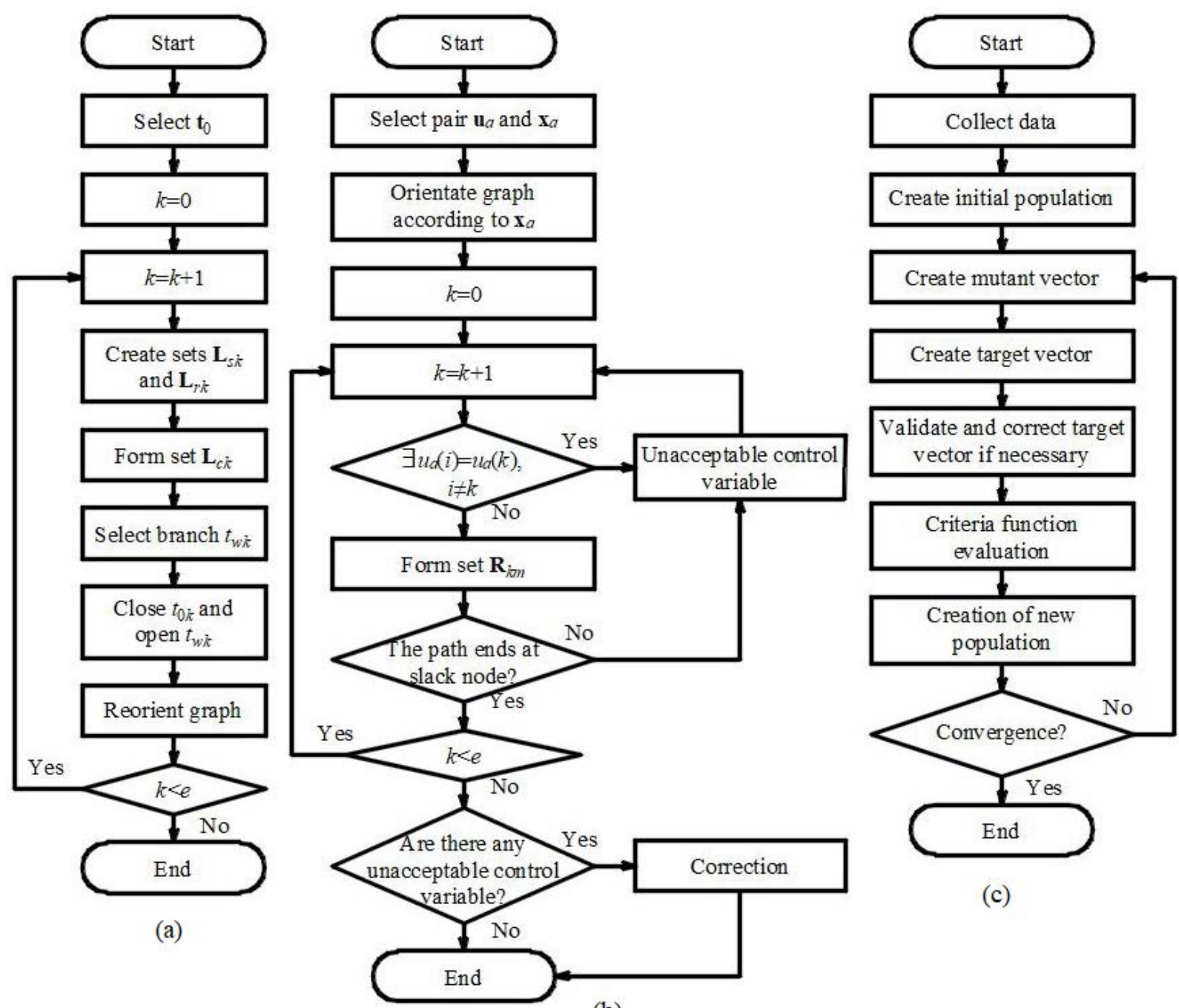

(b)

Fig. 1. Flowchart of the algorithms: (a) - creation of the initial population, (b) - correction of unfeasible solutions, (c) - proposed optimization algorithm

population elements using the adaptive loop concept is described in the following steps:

- Select an arbitrarily feasible solution $\boldsymbol{t}_{0}$ that will serve to initialize the iterative process. A set of possible values of the $k$-th control variable is a set of branch indexes that can be switched off in order to preserve the radial structure when the $\boldsymbol{t}_{0 k}$ switch is closed. This set is labelled with $\boldsymbol{L}_{c k}$, and its formation is described below.

- Add all branch indexes on the path from the sending (receiving) end of the branch $\boldsymbol{t}_{0 k}$, using the operator " $\leftarrow$ ", to the set $\boldsymbol{L}_{s k}\left(\boldsymbol{L}_{r k}\right)$.

- Form a set of candidates $\boldsymbol{L}_{c k}$, using a relation

$$
\boldsymbol{L}_{c k}=\left\{t_{0 k}\right\}+\left(\boldsymbol{L}_{s k} \cup \boldsymbol{L}_{r k}\right) \backslash\left(\boldsymbol{L}_{s k} \cap \boldsymbol{L}_{r k}\right) .
$$

Branches corresponding to this set constitute an adaptive loop.

- Arbitrarily choose one branch of set $\boldsymbol{L}_{c k}$ to be switched off $\left(t_{w k}\right)$.
- Close the $t_{0 k}$ switch, open the $t_{w k}$ switch, and reorient the graph.

- If $k<e$ go back to step 2, otherwise proceed to the next step.

- Assign the vector $\boldsymbol{t}_{0}$ an arbitrarily chosen solution from the already formed initial population and continue the process of creating it.

The described procedure is repeated $N_{p}$ times. Figure 1(a) shows the flowchart of this algorithm. The proposed algorithm for creating the initial population is illustrated on the distribution network shown in Fig. 2(a), with three fundamental loops. Red dashed lines represent the branches with tie switches. In further analysis, it has been assumed that the vector $\boldsymbol{t}_{0}$ consists of the following elements $\boldsymbol{t}_{0}=[13,14,15]$, yellow dashed lines in Fig. 2(b). Assuming that in each branch there is a switching device, three sets of fundamental loops are $\boldsymbol{L}_{1}=[2,3,4,7,13]$, green contour in Fig. 2(b), $\boldsymbol{L}_{2}=$ $[3,4,5,6,10,11,14]$, brown dashed contour in Fig. 2(b), and $\boldsymbol{L}_{3}=[2,7,8,9,10,11,12,15]$, blue dashed contour in 

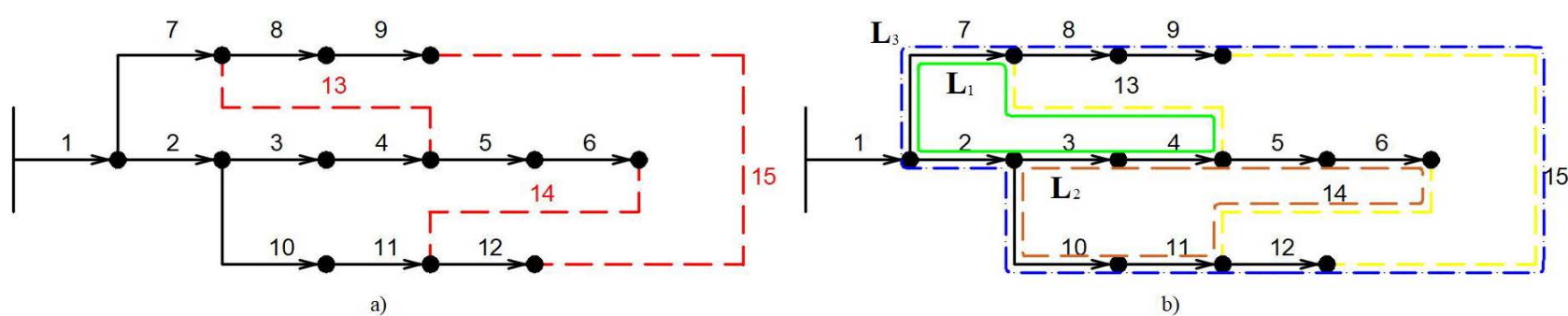

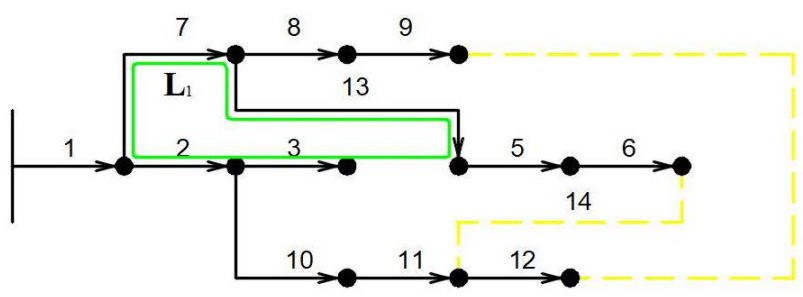

c)

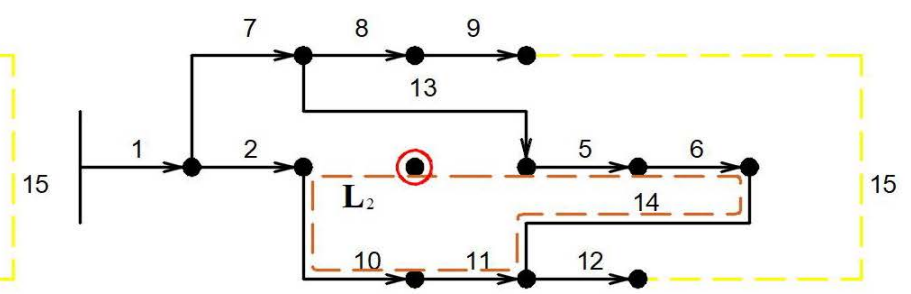

d)

Fig. 2. Fundamental loop concept: (a) - example of distribution network, (b) - fundamental loops; selection of control variables (c) - the first, (d) - the second

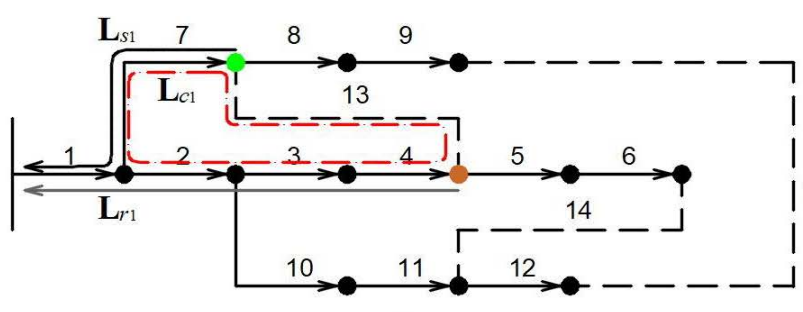

a)

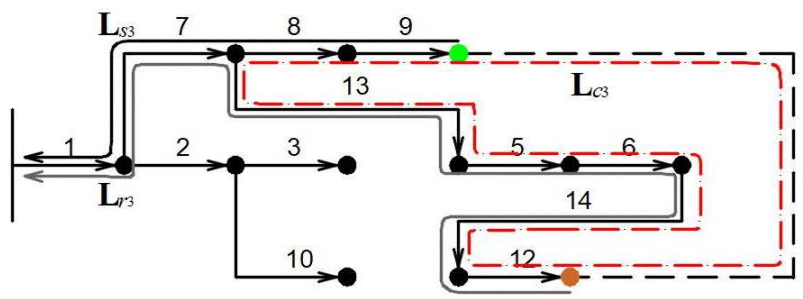

c)

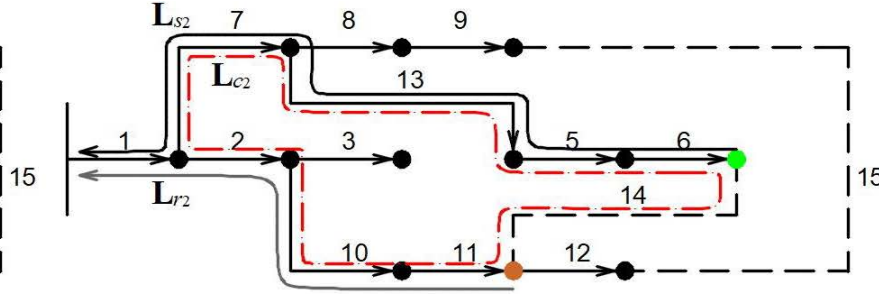

b)

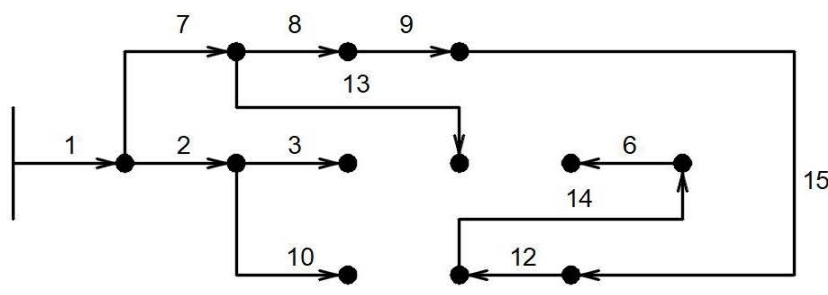

d)

Fig. 3. Creation of the initial population: adaptive loop for control variable (a) - the first, (b) - the second, (c) - the third, and (d) - a feasible solution

Fig. 2(b). It is noted that there are several branches that belong to more than one fundamental loop, and in the case of independent searches double choice of the same branch is not prevented, which leads to forming an unfeasible configuration with unconnected parts of the system. For example, by selecting a third element from the first fundamental loop $\left(L_{13}=4\right)$, Fig. $2(\mathrm{c})$, and the first element from the second fundamental loop $\left(L_{21}=3\right)$, Fig. 2(d), an unfeasible solution is created regardless of the choice of the third control variable, since one node of the network remained unconnected.

When applying the adaptive loop concept let us assume that for the first control variable the same value, as is the case in the previous example, is assigned $\left(t_{w 1}=\right.$ $\left.L_{13}=4\right)$. This means that the switch in branch 13 is closed and the switch in branch 4 is open, Fig. 3(a).
After the reorientation of the graph, for the branch $t_{02}=14$ the path to the supply bus is formed, ie the sets $\boldsymbol{L}_{s 2}$ and $\boldsymbol{L}_{r 2}$ are filled. Since operator " $\leftarrow$ " is used, it is necessary to find the shortest path from both ends of the open branch to the supply bus. Starting from the sending end, green dot in Fig. 3(b), and receiving end, brown dot in Fig. 3(b), the following sets of branches is obtained $\boldsymbol{L}_{s 2}=[6,5,13,7,1]$, black contour in Fig. 3(b), and $\boldsymbol{L}_{r 2}=[11,10,2,1]$, gray contour in Fig. 3(b). Applying (6), a set of allowed values for second control variable $\left(t_{w 2}\right)$ is created, $\boldsymbol{L}_{c 2}=[2,5,6,7,10,11,13,14]$, red dashed contour in Fig. 3(b). In order to preserve the radial structure, it is necessary to close the switch $t_{02}=14$ and open the switch on an arbitrary selected branch from the set $\boldsymbol{L}_{c 2}$. Let the sixth allowed value from the $\boldsymbol{L}_{c 2}$ be selected for the second control variable (by random 
selection), ie $t_{w 2}=11$. After the reorientation of the graph, Fig. 3(c), the described procedure should be repeated for the last control variable. The elements of the set $\boldsymbol{L}_{s 3}$ are $[9,8,7,1]$, while the elements of the set $\boldsymbol{L}_{r 3}$ are $[12,14,6,5,13,7,1]$. Applying $(6)$, a set $\boldsymbol{L}_{c 3}$, the elements of which are $[5,6,8,9,12,13,14,15]$, is created. Closing the switch in branch number 15 and selecting any allowed value for the third control variable, a connected radial network is created at which further optimization calculations can be made. Assume that the value of 1 is obtained using a random number generator. In this case, the vector of the control variables has the following elements $\boldsymbol{t}_{a}=[4,11,5]$, Fig. 3(d).

As has been shown, it is necessary to create a new set of allowed elements for each control variable while accessing adaptive loops. This additional search slows down the process of creating the initial population, but time savings are achieved by making each created solution feasible. Using the fundamental loop concept, the initial process will be repeated more times compared to the proposed concept because there is no possibility of preventing the creation of a solution that does not have a radial structure.

\subsection{Correction of unfeasible solutions}

Operator rules of selected meta-heuristic algorithm can create an unfeasible solution. In order to avoid the rejection of such solution, it is necessary to correct it, while, simultaneously, it is desirable to keep as many components of that solution as possible. Before correction, it is necessary to determine the "good" and "bad" control variables of the observed vector. The adaptive loop concept can be used for this purpose.

Suppose it is necessary to check the feasibility of the vector $\mathbf{u}_{a}=\left[u_{a 1}, \ldots, u_{a e}\right]$. The parent of the generated vector, $\mathbf{x}_{a}$, is used to verify the feasibility of the vector $\mathbf{u}_{a}$. The orientation of the graph is based on the base state, $\boldsymbol{u}_{0}=\mathbf{x}_{a}$. The iterative procedure involves a successive analysis of each control variable.

(1) If the $k$-th control variable is identical to an already considered one in the same vector, such a control variable is declared unacceptable and its replacement is required, otherwise proceed to the next step.

(2) Using the operator " $\rightarrow$ " the $\boldsymbol{R}_{k m}$ set is filled with downstream branches starting from receiving end of disconnected branch. In this case, due to branching of the network, there are several possible paths that need to be checked:

- If at the end of any path, obtained by searching with the operator " $\rightarrow$ ", there is a branch corresponding to a control variable $j$ from the vector $\boldsymbol{u}_{0}$, the search process continues from the branch $u_{0 j}$ using the operator " $\leftarrow$ ". If any such formed path ends in the supply bus of the network, the control variable is declared acceptable, otherwise such variable is declared unacceptable. Whenever a decision variable is confirmed by the adaptive loop concept as acceptable, it is necessary to open the switch $\boldsymbol{u}_{a k}$ and close the switch $\mathbf{u}_{0 j}$, and the new orientation of the graph should be determined.

- If the ends of the downstream paths, created by the operator " $\rightarrow$ ", does not continue to one of the branches defined by the control variables of vector $\mathbf{u}_{0}$, such variable is declared unacceptable.

(3) If $k<e$ go back to step 1.

(4) In the case of unacceptable variables, replace them with acceptable solutions using the procedure for creating the initial population. In this procedure, good variable dictates the network configuration from which the repair process begins.

The described process can better be understood by example. Assume that the vector $\boldsymbol{u}_{0}=\mathbf{x}_{0}=[13,14,15]$ is chosen for the parent. Also, assume that the vector $\mathbf{u}_{a}=[3,4,11]$ is created using the optimization operator rules. As can be seen from previous, this combination of branch exclusion is unacceptable. The verification process begins with the first control variable $u_{a 1}=3$. Since in this solution there are no identical variables the verification of each variable is reduced to the use of step 2 of the described algorithm. The path set $\boldsymbol{R}_{k m}$ is filled using the operator " $\rightarrow$ ", where $k$ represents a regular number of control variables and $m$ the number of possible paths. In this case, two paths are created $\boldsymbol{R}_{11}=[4,5,6]$ and $\boldsymbol{R}_{12}=$ [4]. The first path, $\boldsymbol{R}_{11}$, can be continued with a branch $u_{02}=14$, while the second path, $\boldsymbol{R}_{12}$, is continued by the branch $u_{01}=13$. The search process continues from branch $u_{02}$ (arbitrarily selected) using operator " $\leftarrow$ ". The vector $\boldsymbol{R}_{11}$ receives the final form $\boldsymbol{R}_{11}=[4,5,6,14,11,10,2,1]$, Fig. 4(a). Since the path ends in the supply bus, the first control variable is declared acceptable. After closing the switch in branch 14 and opening the switch in branch 3 it is necessary to reorient the graph of the observed distribution network (red arrows with a star indicate a change in the power flow due to the disconnection of the branch), Fig. 4.

After this, the process is continued by verifying second control variable, $u_{a 2}$. By turning off the switches in the branch $u_{a 2}$, it is not possible to form the requested path $\boldsymbol{R}_{21}$, Fig. 4(b). Therefore, the second control variable is declared unacceptable and the verification process is continued by examining the last (third) control variable. Sets obtained using the operator " $\rightarrow$ " are now $\boldsymbol{R}_{31}=$ [14] (the branch $u_{02}=14$ has changed status when checking the first variable), whose path is continued by branch $u_{01}=13$ and $\boldsymbol{R}_{32}=$ [12] which continues with branch $u_{03}=15$. The search process continues from branch $u_{03}$ (arbitrarily selected) using operator " $\leftarrow$ ". The path set $\boldsymbol{R}_{32}$ receives the final form $\boldsymbol{R}_{32}=[12,15,9,8,7,1]$, Fig. 4(c). Since the path ends in the supply bus, this control variable is also declared as acceptable.

After opening the switch in branch 11 with the closing of the switch in branch 15 and the corresponding reorientation of the graph, the control variable $u_{a 2}$ can be corrected. Starting from the ends of branch 13 (in this case corresponding to the only unused variable of vector $\mathbf{u}_{0}$ ), 


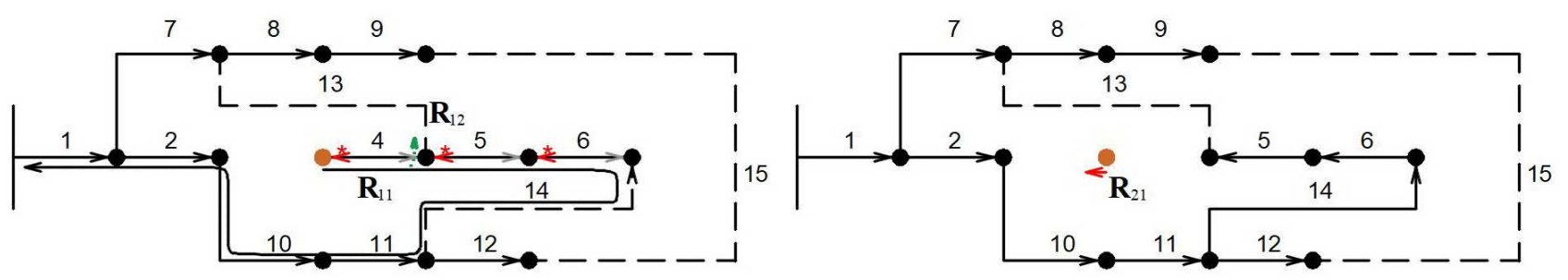

a)

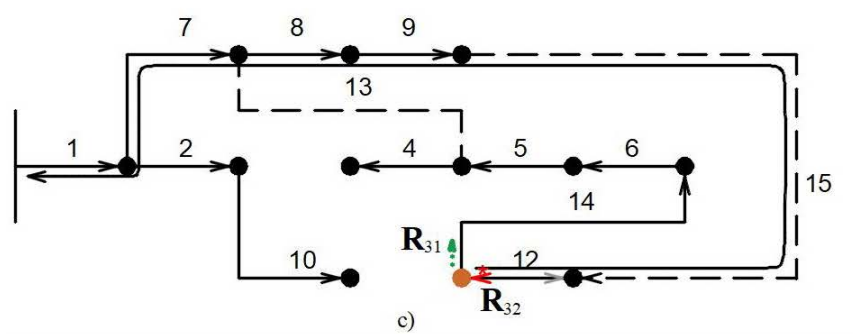

b)

c)

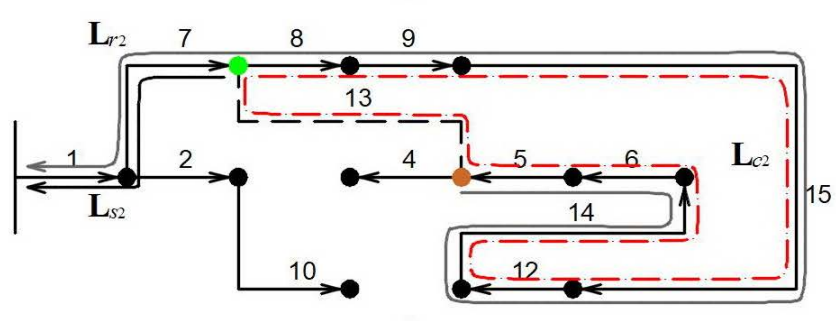

d)

Fig. 4. Validation of generated solution control variables: (a) - the first, (b) - the second, (c) - the third, and (d) - correction of unacceptable control variable

the sets $\boldsymbol{L}_{s 2}=[7,1]$ and $\boldsymbol{L}_{r 2}=[5,6,14,12,15,9,8,7,1]$ are created. Applying (6), a set of allowed candidates for second control variable, $\boldsymbol{L}_{c 2}=[5,6,8,9,12,13,14,15]$, is obtained, Fig. 4(d). The selection of the switch that will be turned off is made using the random number generator. Assume that the value of 2 is obtained using a random number generator. In this case, the vector of the control variables has the following elements $\boldsymbol{u}_{a}=[3,6,11]$.

\section{Optimization algorithm}

The described adaptive loop concept can be applied to any meta-heuristic optimization algorithm. In the proposed optimization algorithm, the distribution network reconfiguration is solved using the $\mathrm{DE}$ algorithm because of its reliability and versatility [24].

Like all evolutionary methods, DE works with a population of vectors that represent potential solutions to the observed problem. This optimization method has only a few control variables (mutation and crossover factors) which can remain fixed throughout the entire optimization procedure. In the context of the problem considered in this paper, it is not possible to apply the classical DE since it works with real numbers. Rather, it is necessary to work with a modified version [26] that works with integers. The basic algorithm operations are initialization, mutation, crossover, selection and evaluation.

The control variables are directly coded as integer values within its corresponding bounds. The initialization process can generate $N_{\mathrm{p}}$, individuals randomly, and should try to cover the entire search space uniformly in the form

$$
\boldsymbol{X}_{p}(0)=\operatorname{round}\left(\boldsymbol{X}_{p}^{L}+r_{m}\left(\boldsymbol{X}_{p}^{U}-\boldsymbol{X}_{p}^{L}\right)\right), p=1, \ldots, N_{p},
$$

where, $r$ is a random number in the range $[0,1], \boldsymbol{X}^{L}$ and $\boldsymbol{X}^{U}$ are lower and upper bound of control variable, and round is a function that rounds the real number to the nearest integer.

A mutant individual is generated according to

$$
\begin{array}{r}
\boldsymbol{V}_{p}(t+1)=\boldsymbol{X}_{r 1}(t)+\operatorname{round}\left(F_{m}\left(\boldsymbol{X}_{r 2}(t)-\boldsymbol{X}_{r 3}(t)\right)\right) \\
p=1, \ldots, N_{p},
\end{array}
$$

where random indices $r_{1}, r_{2}, r_{3}, \in\left[1, \ldots, N_{p}\right]$ are integer and mutually different. The mutation factor, $F_{m}$, is a constant and has to be set by the user.

While the population diversity is small, the candidate individuals will rapidly gather together so that the individuals cannot be further improved, which may result in a premature convergence. Aimed at increasing the local diversity of the mutant individuals, a binomial crossover is introduced. In the crossover operation, each gene of the $i$-th individual is reproduced from the mutant vectors and the current individual, where the crossover factor, $C_{r}$, is a constant and has to be set by the user, and $z_{\text {rand }}$ randomly selected the parameter which might take values from the range $[1, e]$

$$
u_{p z}(t+1)= \begin{cases}x_{p z}(t) & r_{m}>C_{r} \\ v_{p z}(t+1) & r_{m} \leq C_{r} \vee z=z_{\mathrm{rand}}\end{cases}
$$

In DE, each population vector is crossed with a randomly generated mutant vector. Since the current population of vectors already satisfies all bound constraints, only contributions from mutant vectors $\left(\boldsymbol{V}_{p}\right)$ potentially violate parameter limits. Consequently, bounds need to be checked only when a mutant parameter is selected for the target vector $\left(u_{p z}\right)$. In this paper, the bounce-back method for bounds correction was used. This method replaces a vector that has exceeded one or more of its bounds by a valid vector that satisfies all boundary constraints. This strategy takes the progress toward the optimum into account 
by selecting a parameter value that lies between the base parameter value $\left(\boldsymbol{X}_{r 1}\right)$ and the bound being violated. As the population moves toward its bounds, the bounce-back method generates vectors that will be located even closer to the bounds [24].

The evaluation function of an offspring is one-to-one "knockout" comparison to that of its parent. The competition means that any parent will be replaced by its offspring in the next population if the fitness of the parent is worse than that of its offspring, and vice versa. The described procedure continues until the convergence criterion is satisfied.

\section{Algorithm}

The proposed optimization algorithm that includes a new selection of individuals, as well as their verification and correction, in every iteration of the algorithm, can be described by the following steps:

1 Collecting all relevant data and adjusting the parameters of the optimization algorithm.

2 while $p \leq N_{p}$ do

3 Creating the $p$-th vector of the initial population by using the algorithm given in Section 3.1

4 Power flow calculation

5 Constraints verification of control variables

6 if Constraints $=1$ then

7 Criterion function evaluation of the $p$-th vector

$8 p=p+1$

9 end if

\section{0 end while}

11 condition $=1, N_{\text {iteration }}=0$

12 while condition do

$13 N_{\text {iteration }}=N_{\text {iteration }}+1$

14 for $p=1$ to $N_{p}$ do

15 Creation of mutant vectors, $\boldsymbol{V}_{p}$

\section{6 end for}

17 for $p=1$ to $N_{p}$ do

18 Creation of target vectors, $\boldsymbol{U}_{p}$

\section{9 end for}

20 for $p=1$ to $N_{p}$ do

21 Validation of the target vector by using the algorithm given in Section 3.2 if $\boldsymbol{U}_{p}$ unfeasible then

23 Correction of target vector, $\boldsymbol{U}_{p}$, by using the algorithm given in Section 3.2

\section{3 end for}

34 if Convergence $=1$ then

$35 \quad$ condition $=0$

\section{6 end if}

\section{7 end while}

Figure 1(c) shows the flowchart of proposed algorithm. The constraints are considered in two places (rows 6 and 28) in the algorithm. The first constraints validation, (1) - (5), is done in the process of creating an initial population. If any constraint is violated, the if command (rows 6-9) will not be executed, so the counter $p$ will not be increased by one. Only when all constraints are satisfied (line 6 ) such a vector will be remembered at the $p$-th position in the initial population and the counter is increased by one. The second constraints validation is made after changes in control variables that occur as a result of optimization algorithm operators. If one of the constraints, (1)-(5), is violated (line 28) the parent $\boldsymbol{X}_{p}$ will be passed to the next generation, since he is certainly feasible. If all constraints are satisfied (line 30) the offspring, $\boldsymbol{U}_{p}$, will go to the next population only if it is better than the parent, $\boldsymbol{X}_{p}$.

Two conditions were used as the criteria of convergence. The first condition relates to the maximum number of iterations, and for the second convergence criterion the repetition of the best solution was selected. In other words, if the best solution does not change during a predetermined number of iterations, the algorithm stops.

\section{Test results}

In this paper, two cases of distribution network reconfiguration were considered. In the first case, it is assumed that power consumption of all buses is known. This was done in order to compare the proposed algorithm with the fundamental loop concept. Forward/backward sweep method was used for power flow calculations [28]. Due to low observability of the distribution network, consumption in all nodes is not known. However, it is possible to predict the range of power consumption for each node of the network. In recent years, there is a growing presence of renewable energy in distribution systems. Wind turbines are the most commonly used renewable energy sources in distribution systems. Because of the stochastic nature of the wind it is not possible to accurately predict production at any point of time. In the second case, uncertainty in production and consumption is modelled using fuzzy numbers. The uncertainty of the supply voltage was also considered, which is also represented by the fuzzy number ( $V_{1}=0.95 \mathrm{pu}, V_{2}=V_{3}=1 \mathrm{pu}, V_{4}=1.05 \mathrm{pu}$ ), Fig. $5(\mathrm{a})$. For the power of it is assumed that will certainly be higher than $S_{L 1}=85 \%$, most likely lies between $S_{L 2}=90 \%$ and $S_{L 3}=110 \%$, and will be less than $S_{L 4}=115 \%$ of expected consumption, Fig. 5(b). It was assumed that the data from $[29,30]$ represent the expected power of consumption. Production is also shown with a trapezoidal 

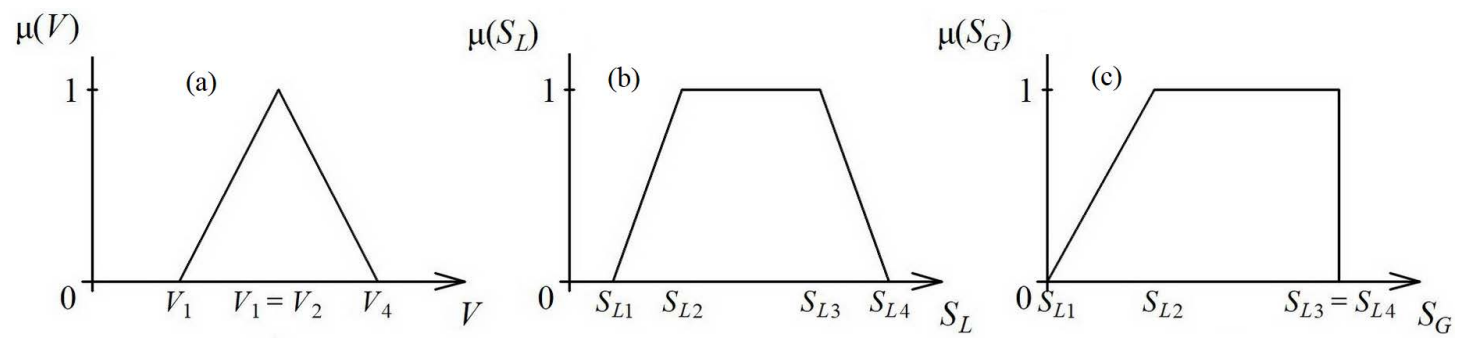

Fig. 5. Fuzzy membership function(a) - bus voltage,(b) - power of consumption,(c) - power of production

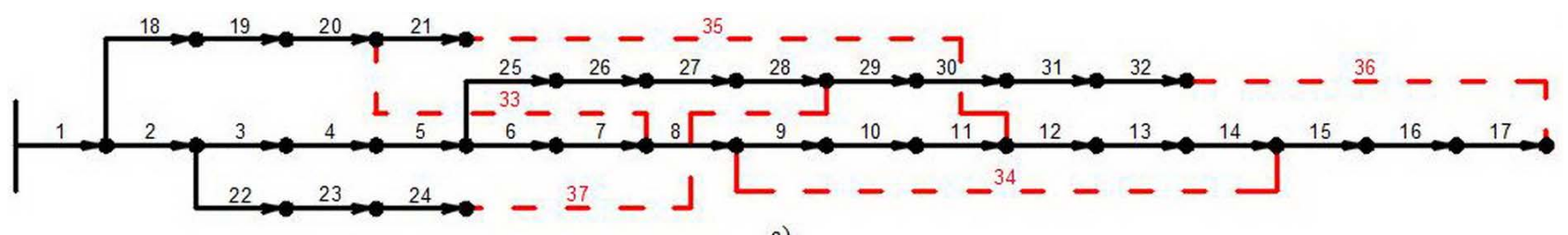

a)

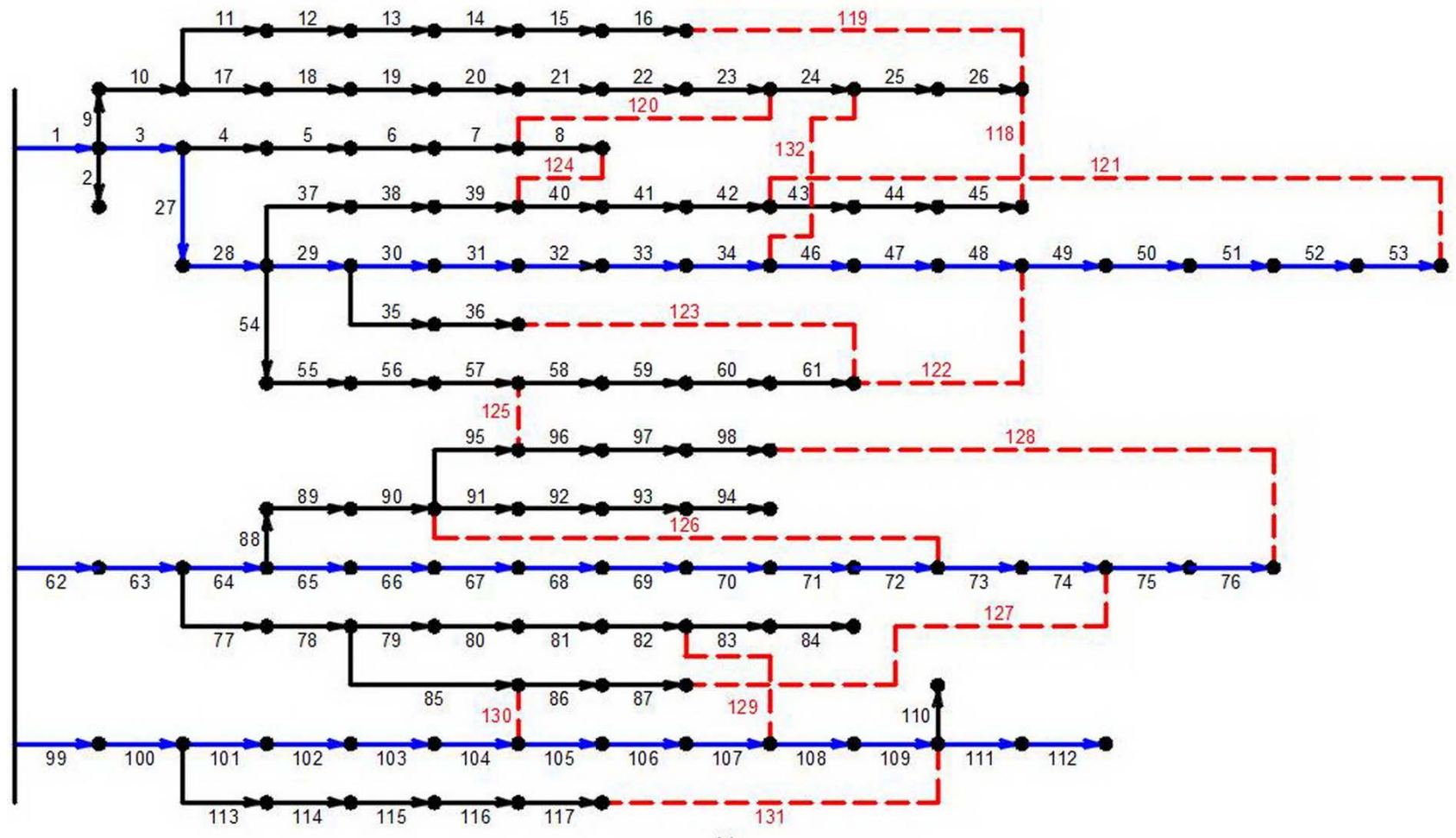

b)

Fig. 6. Test systems:(a) - 33 bus,(b) -119 bus

fuzzy number, Fig. 5(c), where the coordinates are defined by the following set $[0,50,100,100] \%$. For the fuzzy load flow calculation, the previously developed algorithm was used, [23].

All tests were performed on two standard test distribution network, 33 and 119-bus system, which are shown in Fig. 6. Tie branches are marked with red numbers and dashed lines, and for the second test network main stream feeders are marked with blue lines.

In the optimization calculations, the number of population members was $N_{p}=50$. The following values were adopted for optimization algorithm parameters: $F_{m}=0.9, C_{r}=0.8$. For the first convergence criterion, the value of 100 iterations was selected. For the second convergence criterion, a value of 8 iterations was selected.

The first test system has in total 33 buses and 5 tie switches. The total real and reactive demand is $3.715 \mathrm{MW}$ and 2.3 Mvar, respectively. All other necessary data (branch impedance, load, branch current limit) can be found in [29]. For the base state, shown in Fig. 6(a), with supply bus voltage $V_{s b}=12.66 \mathrm{kV}$, the losses in the observed distribution network are $202.68 \mathrm{~kW}$. The time required for the execution of one load flow was $0.01 \mathrm{~s}$.

Table 1 shows the results of the analysis performed on 33 bus network. The first column shows considered cases: FL - Fundamental Loop, AL - Adaptive Loop, 

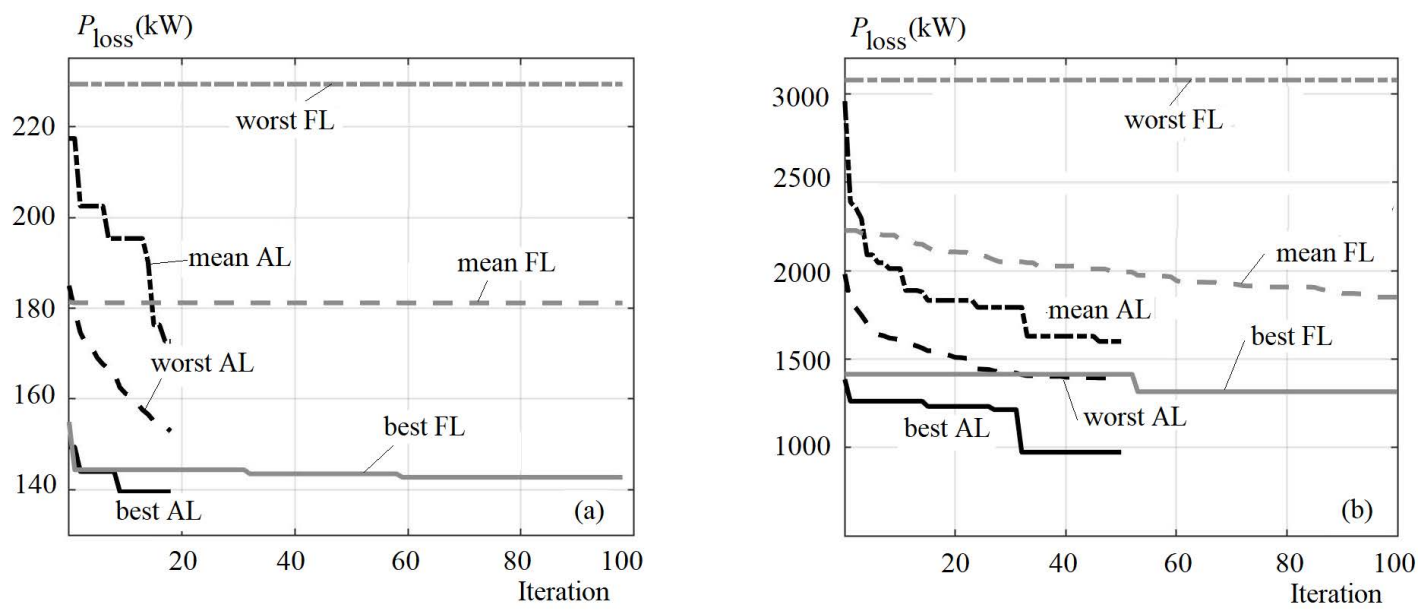

Fig. 7. Comparative analysis of different concepts(a) - 33-bus system, (b) - 119-bus system

Table 1. Results for 33 bus system

\begin{tabular}{lcccccccc}
\hline Case & \multicolumn{4}{c}{ Open branches } & $\begin{array}{c}P_{\text {loss }} \\
(\mathrm{kW})\end{array}$ & NI & $\begin{array}{c}\text { Time } \\
(\mathrm{s})\end{array}$ \\
\hline $\mathrm{FL}$ & 37 & 7 & 34 & 11 & 32 & 142.76 & 98 & 452.8 \\
$\mathrm{AL}$ & 37 & 7 & 14 & 9 & 32 & 139.55 & 18 & 73.4 \\
$\mathrm{ALF}$ & 37 & 7 & 14 & 8 & 36 & 64.51 & 25 & 95.2 \\
\hline
\end{tabular}

ALF - Adaptive Loop Fuzzy. The second column shows the values of control variables, ie the ordinal numbers of branches that should be opened in order to minimize real power losses. The results of the optimization are shown in the third column, while the fourth column shows the required number of iterations of the optimization algorithm. The fifth column shows the execution time of the algorithm.

The results from the first two rows of Table 1 serve to compare the results of the proposed method and the fundamental loop concept when consumption in nodes is known. On the one hand, better solutions are obtained for a significantly smaller number of iterations compared to the case when the fundamental loop concept was applied. Fig. 7(a) show the comparison of results obtained using different concepts for 33-bus system. The solid line shows the change of the best solution during iterations, while the dashed line displays the change of the mean value of the examined criterion function of all population elements. In addition to this, the dash dot line shows the change of the worst solutions during iterations. As can be seen from Fig. 7(a), using the fundamental loop concept, the final solution is achieved in three steps (flat segments in Fig. 7).

By conducting a detailed analysis of the best vector within each population, it was found that they were not constant along the entire segment. However, this change in the control variables of the best vector did not cause major changes in the value of the criterion function, which directly affected the speed of convergence of the entire optimization process.

Power flow calculation is the most demanding process when the fundamental loop concept is applied. The fact is that the load flow calculation for a single configuration takes a very short time, as mentioned earlier, but due to the large number of created solutions related to the rejection of unacceptable solutions, the number of power flow calculation drastically increase. In average, $88 \%$ of the time is spent on a power flow calculation when the fundamental loop concept is used.

The third row in Tab. 1 presents the results of the proposed algorithm when uncertainties in consumption and production are observed. The wind turbines are placed in three buses, receiving ends of the branches 11, 23 and 28 , wherein it is assumed that all generators have the same nominal power (1 MVA), and the same power factor $(p f=0.95)$. Generator production profile is shown in Fig. 5(c). On the one hand, the only difference between the AL and ALF approach is in the power flow calculation that is a bit more complex for a fuzzy approach and requires a longer calculation time. On the other hand, smaller losses of real power were obtained due to the presence of distributed generators.

The second test system has in total 119 buses and 15 tie switches. The total real and reactive demand is 22.71 MW and 17.04 Mvar, respectively. Branch impedance and load data for calculation of the observed criteria function for the 119-bus test distribution network can be found in [30]. It is assumed that main stream feeder (blue line) can be loaded with a maximum current of $750 \mathrm{~A}$, while the laterals can be loaded with a maximum current of $400 \mathrm{~A}$. The losses in the observed distribution network are $1.31 \mathrm{MW}$, and this applies for the base state, which is shown in Fig. 6(b), with supply bus voltage $V_{s b}=11 \mathrm{kV}$. The time required for the execution of one load flow is $0.05 \mathrm{~s}$

The first two rows of Tab. 2 show the results for both concept, the fundamental loop and the adaptive loop, for 

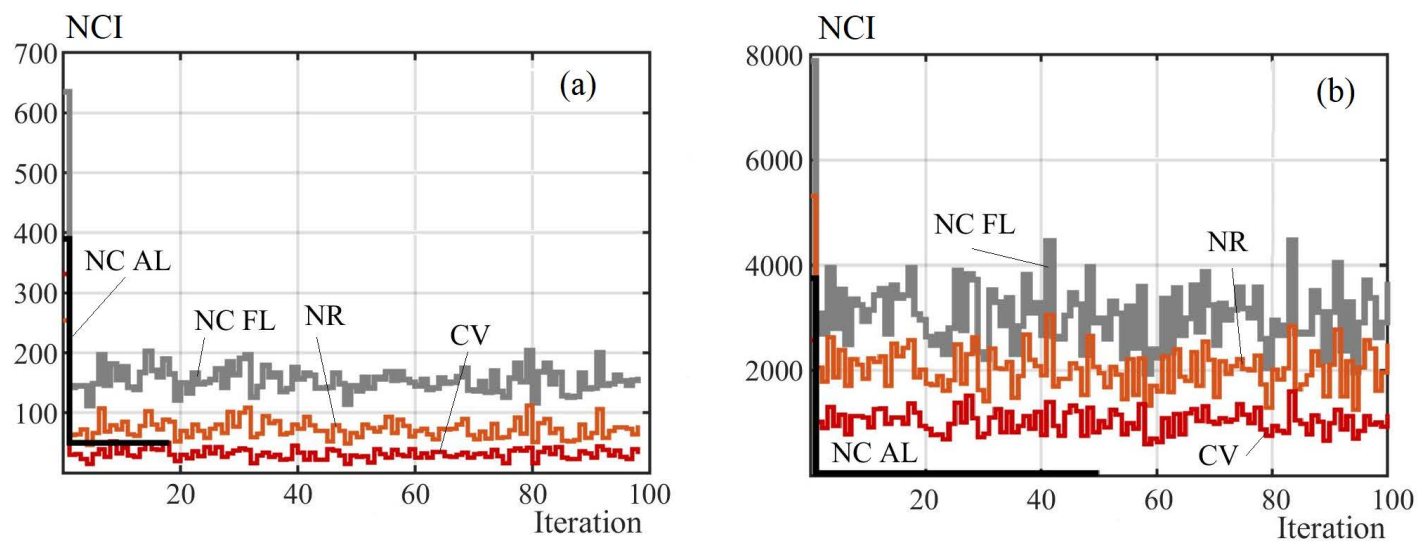

Fig. 8. Number of created individuals:(a) -33 bus,(b) -119 bus

Table 2. Results for 119 bus system

\begin{tabular}{lllllllllllllllllll}
\hline Case & \multicolumn{11}{c}{ Open branches } & \multicolumn{11}{c}{$P_{\text {loss }}(\mathrm{kW})$} & NI & Time (s) \\
\hline FL & 9 & 23 & 44 & 38 & 54 & 47 & 41 & 60 & 66 & 85 & 126 & 80 & 62 & 102 & 5 & 1315.76 & 100 & 6312.8 \\
$\mathrm{AL}$ & 42 & 26 & 23 & 51 & 122 & 58 & 39 & 95 & 71 & 74 & 97 & 129 & 130 & 109 & 34 & 912.43 & 50 & 1283.4 \\
$\mathrm{ALF}$ & 42 & 26 & 22 & 50 & 122 & 60 & 39 & 125 & 126 & 70 & 76 & 129 & 130 & 109 & 34 & 659.08 & 62 & 1747.6 \\
\hline
\end{tabular}

the 119-bus test system. Because of the complexity of the problem with 15 control variables, the fundamental loop concept has converged after reaching the maximum number of iterations. As shown in the second row (AL) of Tab. 2, better solutions for a smaller number of iterations were found for this test network using the adaptive loop concept.

By applying the adaptive loop concept, the calculation time increases proportionally with increasing dimensionality of the problem. Moreover, the total duration of the calculation is significantly higher compared to the proposed concept due to the greater dimensionality of the problem and the difficulty of forming feasible solutions using the fundamental loop concept. As can be seen from Fig. 7, the presented curves in the fundamental loop concept for the worst solution and for the mean value of the criterion function of the entire population were slightly changed during the entire optimization process. This means that a large number of generated solutions were worse than existing ones, $i e$ the population changed very slowly during iterations. On the other hand, this is not the case when the concept of adaptive loops is applied.

The results shown in the third row of Tab. 2 refer to the proposed algorithm when uncertainties in consumption and production are observed. Three wind turbines are placed in system, receiving ends of the branches 71,73 and 110, wherein it is assumed that all generators have the same nominal power (1MVA), and the same power factor $(p f=0.95)$. In this case, smaller losses of real power were obtained due to the presence of distributed production.
The results shown in Tab. 1 and 2 point out that using the fundamental loop concept with only one base state, which is most often applied in the literature [7,9-11,17], does not perform a complete search of the entire solution space and the obtained solution represents a local optimum. With the adaptive loop concept, this search is expanding, and better solutions were found. Another advantage of the adaptive loop concept is a smaller number of iterations, with a shorter computation time.

Significantly more solutions are formed when the fundamental loop concept is used. Fig. 8 shows the total number of individuals created in each population using both concepts in the case when the reduction of real power losses is considered for both test systems. The largest number of generated vectors occurs during the formation of the initial population in the context of both concepts. Nevertheless, when the adaptive loop concept is used, this number is almost two times smaller. The reason for this improvement is the fact that using the adaptive loop concept only radial configuration can be created, while this is not the case with fundamental loop concept. On the other hand, there is no possibility of preventing the creation of solutions that would violate operational constraints related to voltages, limitation of branch load, and so, the number of these solutions is approximately identical in the initial population regardless of the chosen concept.

The results of the proposed algorithm are compared with the solutions found in the literature. The nominal value of the supply bus voltage is assumed for the verification purposes of all solutions. Table 3 shows the results of the comparison. The data from the fourth column of 
Table 3. Comparison results for test distribution systems

\begin{tabular}{|c|c|c|c|c|c|c|c|c|c|c|c|c|c|c|c|c|c|}
\hline \multirow{2}{*}{$\frac{\text { Test }}{33 \mathrm{Bus}}$} & \multirow{2}{*}{$\begin{array}{c}\text { Method } \\
\text { Proposed method }\end{array}$} & \multirow{2}{*}{$\frac{\text { Ploss }(\mathrm{kW})}{139.55}$} & \multicolumn{15}{|c|}{ Open branches } \\
\hline & & & 37 & 7 & 14 & 9 & 32 & & & & & & & & & & \\
\hline & Ahmadi (31) & 139.98 & 7 & 9 & 14 & 28 & 32 & & & & & & & & & & \\
\hline & Souza (32) & 139.55 & 7 & 9 & 14 & 32 & 37 & & & & & & & & & & \\
\hline & Baran (28) & 182.21 & 11 & 31 & 28 & 35 & 33 & & & & & & & & & & \\
\hline & de Oliveira (33) & 139.55 & 7 & 9 & 14 & 32 & 37 & & & & & & & & & & \\
\hline & Duan (34) & 139.55 & 7 & 9 & 14 & 32 & 37 & & & & & & & & & & \\
\hline & Mendoza (20) & 139.55 & 7 & 9 & 14 & 32 & 37 & & & & & & & & & & \\
\hline \multirow[t]{4}{*}{119 Bus } & Proposed method & 912.43 & 42 & 26 & 23 & 51 & 122 & 58 & 39 & 95 & 71 & 74 & 97 & 129 & 130 & 109 & 34 \\
\hline & Srinivasa Rao (35) & 1091.40 & 42 & 26 & 22 & 52 & 122 & 61 & 124 & 125 & 74 & 71 & 128 & 129 & 130 & 131 & 32 \\
\hline & Zhang (30) & 912.43 & 42 & 26 & 23 & 51 & 122 & 58 & 39 & 95 & 74 & 71 & 97 & 129 & 130 & 109 & 34 \\
\hline & Abdelaziz (36) & 920.05 & 23 & 26 & 33 & 39 & 42 & 51 & 58 & 71 & 74 & 95 & 97 & 109 & 122 & 129 & 130 \\
\hline
\end{tabular}

Tab. 3, which are taken from the references, are adapted to the numbering shown in Fig. 6(b).

Better speed in finding the optimal solution in the case of adaptive loop concept is a direct consequence of the proposed algorithm for correction of unfeasible solutions. If the operation of selected optimization algorithm produces an unfeasible solution in terms of network configuration (unconnected and/or non-radial), it is corrected and should not be discarded. That is why every member of the population is only processed once, which was not the case in the algorithms that used fundamental loop concept or binary coding.

\section{Conclusion}

This paper presents the adaptive loop concept for encoding the distribution network branches in solving the optimal reconfiguration of the distribution network. Following this concept, a permitted set of solutions that can be chosen is successively created for each control variable. Due to this approach, creation of unfeasible solutions (unconnected and non-radial) is disabled. Verification and correction of unfeasible solutions that are created using an optimization algorithm operator rules can be performed by applying the proposed adaptive loop concept. Two standard different test distribution networks were used for testing the characteristics of the proposed concept. The uncertainty of the distributed generators production was considered using a fuzzy numbers. The proposed concept has shown better characteristics regarding the fundamental loop concept for all considered cases. Furthermore, the optimal value of criterion function was found in a shorter time and for a smaller number of iterations by utilizing the proposed concept.

\section{REFERENCES}

[1] J. C. López, M. Lavorato, and J. F. Franco Mj Rider, "Robust Optimization Applied to the Reconfiguration of Distribution
Systems with Reliability Constraints", IET Generation Transmission \& Distribution, vol. 10, no. 4, pp. 917-927, 2016.

[2] P. M. Quevedo, J. Contreras, and M. J. Rider Allahdadian Javad, "Contingency Assessment Network Reconfiguration in Distribution Grids Including Wind Power Energy Storage", IEEE Transactions on Sustainable Energy, vol. 6, no. 4, pp. 1524-1533, 2015.

[3] S. K. Goswami and S. K. Basu, "A New Algorithm for the Reconfiguration of Distribution Feeders for Loss Minimization", IEEE Transactions on Power Delivery, vol. 7, no. 3, pp. 1484-1491, 1992.

[4] D. Shirmohammadi and H. W. Hong, "Reconfiguration of Electric Distribution Networks for Resistive Line Losses Reduction", IEEE Transactions on Power Delivery, vol. 4, no. 3, pp. 1492-1498, 1989.

[5] A. Bayat, "Uniform Voltage Distribution Based Constructive Algorithm for Optimal Reconfiguration of Electric Distribution Networks", Electric Power Systems Research, vol. 104, pp. 146-155, 2013.

[6] N. T. Linh, T. T. Chuong and T. V. Anh, "A Study on the Effect of Distributed Generation of the Reconfiguration of Distribution Networks", Journal Electrical Engineering Technology, vol. 12, no. 4 , pp. $1435-1441,2017$.

[7] Y.-K. Wu, C.-Y. Lee, L.-C. Liu, and S.-H. Tsai, "Study of Reconfiguration for the Distribution System with Distributed Generators", IEEE Transactions on Power Delivery, vol. 25, no. 3, pp. 1678-1685, 2010.

[8] D. Šošić and P. Stefanov, "Multi-Objective Reconfiguration of Distribution Network", Journal of Electrical Engineering, vol. 69, no. 2, pp. 128-137, 2018.

[9] Z. Li, S. Jazebi, and F. León, "Determination of the Optimal Switching Frequency for Distribution System Reconfiguration", IEEE Transactions on Power Delivery, vol. 32, no. 4, pp. 2060-2069, 2017.

10] F. Sayadi, S. Esmaeili, and F. Keynia, "Feeder Reconfiguration Capacitor Allocation in the Presence of Non-Linear Loads using New P-PSO Algorithm", IET Generation Transmission \& Distribution, vol. 10, no. 10, pp. 2316-2326, 2016.

[11] B. Khorshid-ghazani, H. Seyedi, B. Mohammadi-ivatloo, K. Zare, and S. Shargh, "Reconfiguration of Distribution Networks Considering Coordination of the Protective Devices", IET Generation Transmission \& Distribution, , vol. 11, no. 1, pp. 82-92, 2017.

[12] H. Haghighat and B. Zeng, "Distribution System Reconfiguration under Uncertain Load Renewable Generation", IEEE Transactions on Power Systems, vol. 31, no. 4, pp. 2666-2675, 2016. 
[13] A. M. Tahboub, V. R. Pandi, and H. H. Zeineldin, "Distribution System Reconfiguration for Annual Energy Loss Reduction Considering Variable Distributed Generation Profiles", IEEE Transactions on Power Delivery, vol. 30, no. 4, pp. 1677-1685, 2015.

[14] A. Asrari, S. Lotfifard, and M. Ansari, "Reconfiguration of Smart Distribution Systems with Time Varying Loads using Parallel Computing", IEEE Transactions on Smart Grid, vol. 7, no. 6, pp. $2713-2723,2016$.

[15] H. R. Esmaeilian and R. Fadaeinedjad, "Energy Loss Minimization in Distribution Systems Utilizing an Enhanced Reconfiguration Method Integrating Distributed Generation", IEEE Systems Journal, vol. 9, no. 4, pp. 1430-1439, 2015.

[16] T. Ding, Y. Lin, Z. Bie, and C. Chen, "A Resilient Microgrid Formation Strategy for Load Restoration Considering Master-Slave Distributed Generators Topology Reconfiguration", Applied Energy, vol. 199, pp. 205-216, 2017.

[17] A. M. Eldurssi and R. M. O'Connell, "A Fast Nondominated Sorting Guided Genetic Algorithm for Multi-Objective Power Distribution System Reconfiguration Problem", IEEE Transactions on Power Systems, vol. 30, no. 2, pp. 593-601, 2015.

[18] A. Asrari and S. Lotfifard Ms Payam, "Pareto Dominance-Based Multiobjective Optimization Method for Distribution Network Reconfiguration", IEEE Transactions on Smart Grid, vol. 7, no. 3, pp. 1401-1410, 2016.

[19] E. Mahboubi-Moghaddam, M. R. Narimani, M. H. Khooban, A. Azizivahed, and M. J. Sharific, "Multi-Objective Distribution Feeder Reconfiguration to Improve Transient Stability", Electrical Power Energy Systems, vol. 76, pp. 35-43, 2016.

[20] J. Mendoza, R. López, D. Morales, E. Lopez, P. Dessante, and R. Moraga, "Minimal Loss Reconfiguration using Genetic Algorithms with Restricted Population Addressed Operators: Real Application", IEEE Transactions on Power Systems vol. 21, no, 2, pp. 948-954, 2006.

[21] Y.-C. Huang, "Enhanced Genetic Algorithm-Based Fuzzy Multi-Objective Approach to Distribution Network Reconfiguration", IEE Proceedings-Generation Transmission Distribution, vol. 149, no. 5, pp. 615-620, 2002.

[22] A. Sallam, O. Malik, Electric Distribution Systems, 1st ed, A John Wiley \& Sons, 2011.

[23] D. Šošić, M. žarković, and G. Dobrić, "Fuzzy-based Monte Carlo simulation for harmonic load flow in distribution networks, IET Generation Transmission \& Distribution, vol. 9, no. 3, pp. 267-275, 2015.

[24] K. Price, R. Storm, J. Lampinen, Differential Evolution - A Practical Approach to Global Optimization, 1st ed, SpringerVerlag Berlin Heidelberg, 2005,.

[25] D. Zou, S. Li G-g Wang, Z. Li, and H. Ouyang, "An Improved Differential Evolution Algorithm for the Economic Load Dispatch Problems with or without Valve-Point Effects", Applied Energy, vol. 181, pp. 375-390, 2016.

[26] J. Krstivojević, D. Šošić, and A. Savić, "Coordination of Directional Overcurrent Relays by Using a Heuristic Optimization Method", presented at the 2016 Int. Conf. Mediterranean Conference on Power Generation Transmission, Distribution Energy Conversion, Belgrade, Serbia, 6-9 November 2016.
[27] S. Civanlar, J. J. Grainger, H. Yin, and S. H. H. Lee, "Distribution Feeder Reconfiguration for Loss Reduction", IEEE Transactions on Power Delivery, vol. 3, no. 3, pp. 1217-1223, 1988.

[28] D. Shirmohammadi, H. Hong, A. Samlyen, and G. X. Luo, "A Compensation-Based Power Flow Method for Weakly Meshed Distribution Transmission Networks", IEEE Transactions on Power Systems, vol. 3, no. 2, pp. 753-762, 1988.

[29] M. Baran and F. Wu, "Network Reconfiguration in Distribution Systems for loss Reduction Load Balancing", IEEE Transactions on Power Delivery, vol. 4, no. 2, pp. 1401-1407, 1989.

[30] D. Zhang, Z. Fu, and L. Zhang, "An Improved TS Algorithm for Loss-Minimum Reconfiguration in Large-Scale Distribution Systems", Electric Power Systems Research, vol. 77, no. 5-6, pp. 685-694, 2007.

31] H. Ahmadi and J. R. Martí, "Minimum-Loss Network Reconfiguration: A Minimum Spanning Tree Problem", Sustainable Energy Grids Networks, vol. 1, pp. 1-9, 2015.

32] S. S. Souza, R. Romero, and J. F. Franco, "Artificial Immune Networks Copt-aiNet Opt-aiNet Applied to the Reconfiguration Problem of Radial Electrical Distribution Systems", Electric Power Systems Research, vol. 119, pp. 304-312, 2015.

33] L. de Oliveira, E. de Oliveira, F. Gomes, I. Silva, A. Marcato, and P. Resende, "Artificial Immune Systems Applied to the Reconfiguration of Electrical Power Distribution Networks for Energy Loss Minimization", Electrical Power Energy Systems, vol. 56, pp. 64-74, 2014.

[34] D.-L. Duan X.-L. Ling, $\mathrm{X}_{i}-\mathrm{Y}$. Wu and B. Zhong, "Reconfiguration of Distribution Network for Loss Reduction Reliability Improvement based on an Enhanced Genetic Algorithm", Electrical Power Energy Systems, vol. 64, pp. 88-95, 2015.

35] R. Srinivasa Rao, S. Narasimham, M. Ramalinga Raju, and A. Srinivasa Rao, "Optimal Network Reconfiguration of LargeScale Distribution System Using Harmony Search Algorithm", IEEE Transactions on Power Systems, vol. 26, no. 3, pp. 1080 $-1088,2011$.

36] A. Abdelaziz, F. Mohamed, S. Mekhamer, and M. Badr, "Distribution System Reconfiguration using a Modified Tabu Search Algorithm, Electric Power Systems Research, vol. 80, no. 8, pp. $943-953,2010$.

Received 6 June 2019

Darko Šošić was born in Serbia in 1984. He received his BSc and MSc PhD degree in 2007, 2009 and 2015 from the School of Electrical Engineering, University of Belgrade, Serbia. His main research interests include power system analysis, distribution system analysis, renewable power production and modeling. Currently, he is an assistant professor with the University of Belgrade - School of Electrical Engineering, Serbia.

Predrag Stefanov was born in Serbia in 1964. He received his BSc, MSc and PhD degree in 1988, 1995 and 2004 from the School of Electrical Engineering, University of Belgrade, Serbia. His main research interests are in the field of analysis, exploitation, planning and regulation of power systems. Currently, he is an assistant professor with the University of Belgrade - School of Electrical Engineering, Serbia. 